\title{
AUTO-REDUCTION 'EN MASSE' OF AN INGUINAL HERNIA
}

\author{
By A. Henry Millard, B.Sc., M.B., B.S., F.R.C.S. \\ Late Senior Surgical Registrar, United Cardiff Hospitals
}

It is thought that auto-reduction en masse of an inguinal hernia is a sufficiently rare occurrence to justify the report of another case. Pearce (I93I) estimated the incidence as $I$ in 13,000 of inguinal herniae and found that unless the sac was ruptured (4 per cent.), strangulation always occurred in the pro-peritoneal position. Many authors have noted the apparent latent interval between the time of 'reduction' of the hernia and the onset of further symptoms but no satisfactory explanation of this phenomenon appears to have been advanced to date. Grey Turner (1903-04) reported 'several months' elapsing between apparent reduction and the appearance of symptoms, Walsham (I.90I) two months, Wolfe (I939) two months, Pearce (193I) three weeks, Chapple (I950) five days, and Bailie (I953) three days.

\section{Case Report}

F.G.C., a railway parcel porter, aged 55, was admitted to hospital at 3 p.m. on February 3, 1950, with a history of sudden onset of severe pain in the centre of the abdomen whilst he was lifting some iron bars at work at II.30 p.m. on February 2, 1950. This pain, which was described as 'colicky,' was replaced after about ten minutes by a persistent intense pain in the left lower abdomen. Vomiting had occurred several times and nausea was continuous. A constipated stool had been passed at 5.30 p.m. the previous evening and there were no urinary symptoms.

There was a previous history of a left inguinal hernia for the last two years and the patient was awaiting an operation for its cure. The hernia was easily controlled by a truss which he had worn since it was first noticed. It had not 'been down' for ' three to four weeks' and then had not given any trouble and had gone back without the slightest difficulty.

On examination, the general condition of the patient was good: temp. $97.6^{\circ} \mathrm{F}$., pulse 80 , respiration 20 , B.P. $130 / 85$. The tongue was clean and moist and the abdomen was scaphoid. Palpation revealed very marked tenderness and rigidity in the left iliac fossa immediately above Poupart's ligament. There was a suspicion of a small underlying mass, but in view of the rigidity it was not possible to be more certain of this. A well-marked ' rebound phenomenon' was elicited over the site of tenderness, but no hernia was either visible or palpable and none appeared on coughing. On auscultation the abdomen was completely silent. Rectal examination revealed some indefinite tenderness anteriorly; urine microscopy was negative and there was no loin tenderness. Straight X-ray of the abdomen showed no abnormality. A provisional diagnosis of intestinal strangulation-possibly an auto-reduction en masse of an inguinal hernia, was made and laparotomy performed forthwith. A stomach tube was passed and an I-V saline drip set up preoperatively.

Operation (A.H.M., 5 p.m., February 3, I950)

Under general anaesthesia a right paramedian incision was made and a left inguinal sac containing a coil of small bowel was found, the latter lying in the pro-peritoneal space. Both the entering and returning coil of gut for about 6 in. ( $15 \mathrm{~cm}$.) was collapsed and in spasm, whilst the remainder of the bowel was normal and free from any distension. The hernia was reduced without any difficulty by gentle traction on the gut when a few millilitres of blood-stained fluid escaped from the neck of the sac. The bowel was very congested and cyanotic, but it soon regained its normal colour and was confidently judged to be viable. The neck of the sac easily admitted the tip of the index finger which could be passed through an equally generous internal ring into the inguinal canal. In view of this, after closing the abdominal incision, left inguinal herniorrhaphy was performed via the usual approach. The sac was about $2 \frac{1}{2}$ in. $(6 \mathrm{~cm}$.) long and a little oedematous.

Post-operative convalescence was uneventful and both wounds healed per primum, the patient being discharged symftom-free on February 12, I950.

\section{Discussion}

Casten and Bodenheimer (I94I) state that reduction en masse can occur only if the internal ring is lax and the neck of the sac constitutes an 
unyielding constriction. They consider that if both the internal ring and the neck of the sac are tight, no reduction at all will be possible, whereas if neither are very tight complete reduction will occur.

Pearce (I93I) reported on 193 cases, of which I Io were analysed. In 102 (92.8 per cent.) the sac was displaced into the pro-peritoneal position of which 85 ( 77.3 per cent.) the entire mass was outside the inguinal canal, whilst in the remaining I7 (I 5.5 per cent.) an inguino-pro-peritoneal sac was present with the strangulated viscus in the pro-peritoneal position but the sac still in the inguinal canal. In 8 cases $(7.2$ per cent.) false reduction had occurred by rupture of the sac and displacement only of the strangulated viscus. Rupture of the sac occurred in only 4 per cent. of the whole series. According to Pearce one of three conditions are found:

I. Displacement of an inguinal pro-peritoneal sac plus content to the pro-peritoneal position (most common).

2. Displacement of a purely inguinal sac plus content to the pro-peritoneal position.

3. Displacement of the contents only from an inguinal to a pro-peritoneal position.

He concluded that a preformed pro-peritoneal sac or diverticulum was present in many cases.

It would appear, therefore, that for reduction en masse to occur, three conditions must be fulfilled, viz.:

(a) A lax internal ring.

(b) A relatively narrow neck.

(c) A real or potential pro-peritoneal sac or diverticulum.

However, to date no explanation appears to have been advanced for the latent interval which often occurs between the time of reduction of the hernia and the appearance of further symptoms. A theory is advanced that in these cases reduction is always incomplete and that a coil of bowel remains unobstructed in the pro-peritoneal space where by keeping open the neck of the sac it facilitates the entry of further bowel if circumstances permit. Any sudden increase in intraabdominal pressure, e.g. coughing or straining or perhaps involuntarily, will have the effect of forcing more gut into the pro-peritoneal sac where it becomes jammed at its neck, thus precipitating strangulation. This is much more liable to occur if a truss is worn, since by pressure over the internal ring it tends to prevent any extra bowel which enters the sac from passing through into the inguinal canal.

Furthermore, when considering the mechanics of the primary ' reduction,' one might add a rider to the hypothesis of Casten and Bodenheimer to the effect that once the gut has been reduced beyond the internal ring, it is the size of the neck which determines not only whether any strangulation is relieved but also whether it is likely to recur after a latent interval.

I. If the neck of the sac is large and approximates to that of a lax internal ring, all of the bowel will be reduced.

2. If the neck is very tight, none of the bowel will be reduced and any strangulation will persist unchanged.

3. If the neck of the sac is intermediate in size between $I$ in. and 2 in., sufficient bowel will be reduced to relieve any strangulation, but some will be retained unobstructed in the pro-peritoneal sac. In this event, a latent interval will elapse before more gut is forced into the sac and strangulation supervenes. Although it is conceivable that if all the bowel is reduced it might re-enter the empty pro-peritoneal sac at some future date, the chance of this occurrence would appear to be remote when compared with that which obtains if part of the sac content is retained.

Whilst not challenging Pearce's conclusion that a pre-formed pro-peritoneal sac is present in many cases, it would seem equally likely that the pro-peritoneal sac is artificially produced as the direct result of the force used to attempt reduction which creates the sac by stripping the peritoneum off the deep aspect of the lower anterior abdominal wall.

Finally, it is noteworthy that in this case the occurrence of ' spastic ileus' presented the picture of a 'silent abdomen' in the presence of a dangerous obstructing lesion. In spite of the normal X-ray appearances, the history and clinical features were such that it was possible to make a reasonably confident diagnosis of bowel strangulation pre-operatively and before the onset of later and more ominous signs.

\section{Summary}

I. A case of auto-reduction en masse of an inguinal hernia associated with spastic ileus and with a latent interval of three to four weeks is reported.

2. The frequency of the latent interval is referred to and a theory advanced to explain it.

\section{Acknowledgment}

My thanks are due to Mr. D. B. Foster, Consultant Surgeon, United Cardiff Hospitals, for permission to publish this case.

\section{BIBLIOGRAPHY}

BAILIE, R. N. (1953), Post. med. F., 29, 323.

CASTEN, D., and BỎDENHEIMËR, M. (194I), Surg. 9, 560. CHAPPLE, C. F. (1950), Brit. med. F., i, 286.

PEARCE, H. E. (I93I), Surg. Gynec. Öbstet., 53, 822 .

TURNER, G. GREY (1903-4), Northumberland and Durham med. $\%$.,

WALSHAM, W. J. (1901), Brit. med. $\mathcal{F}$., i, 691 .

WOLFE, H.' R. I. (1939), Brit. F. Surg., 27, 421. 